\title{
Extending AUV Response Robot Capabilities to Solve Standardized Test Methods
}

\author{
Bence Takács ${ }^{1,3}$, Roland Dóczi ${ }^{1}$, Balázs Sütö ${ }^{2}$, János Kalló ${ }^{1}$, \\ Teréz Anna Várkonyi ${ }^{1,3}$, Tamás Haidegger ${ }^{1,3}$, Miklós \\ Kozlovszky ${ }^{2,4}$ \\ ${ }^{1}$ John von Neumann Faculty of Informatics, Óbuda University, Budapest, \\ Hungary \\ ${ }^{2}$ BioTech Research Center, Óbuda University, Budapest, Hungary \\ ${ }^{3}$ Antal Bejczy Center for Intelligent Robotics, Óbuda University, Budapest, \\ Hungary \\ ${ }^{4}$ MTA SZTAKI/Laboratory of Parallel and Distributed Computing, Budapest, \\ Hungary \\ bence.takacs@irob.uni-obuda.hu; suto.balazs@biotech.uni-obuda.hu; \\ teri.varkonyi@irob.uni-obuda.hu; haidegger@irob.uni-obuda.hu; \\ kozlovszky.miklos@nik.uni-obuda.hu
}

\begin{abstract}
Autonomous Underwater Vehicle $(A U V)$ response robots are special multipurpose devices, capable of moving and performing various tasks in water, autonomously, or with human teleoperation. Capability assessment of such devices is hard and complex work. This paper describes our work in AUV Response Robot testing from two aspects: First, additional testing methods are proposed for AUV capability assessment and second, we describe, in detail, how an AUV can be enhanced to pass the existing underwater response robot tests, defined by National Institute of Standards and Technology (NIST). In the first part of the paper, a short overview of the existing AUV testing methods is given, followed by our proposed, new test scenarios. The second part covers a general overview about our system design and development, which enabled the custom, enhanced AUV to pass the test scenarios.
\end{abstract}

Keywords: autonomous underwater vehicles (AUV); response robotics; AUV testing; underwater manipulation; underwater teleoperation

\section{Introduction}

The field of Autonomous Robotics Research has increased tremendously, in popularity, over the last decade, for air, land and sea applications. Emergency response, Autonomous Underwater Vehicles (AUVs), can be equipped with a vast number of sensors and actuators, to be used for a broad range of applications. 
Civilian and military use cases include, underwater mapping, structural inspection of power plants, localization of leaking underwater pipes or finding missing people after an accident. For these tasks, underwater navigation is essential. In most cases, CCD cameras are employed in the visual domain as sensors. Nevertheless, underwater conditions make it difficult to use normal camera systems with RGB color space for object detection. Additional problems arise from disturbances originated from the external environment, such as underwater lighting, reflection and ray scattering, high pressure and last, but not least, high conductivity of liquids [1].

\subsection{Motivation}

The motivation behind the research is twofold. First, there is the social drive: our research is mainly inspired by the need to remedy the consequences of industrial accidents (e.g., the Fukushima Daiichi accident in 2011). It is often required that underwater robots survey the scene, collect environmental data and to identify critical hazards. Such scenarios require complex task execution, realized through autonomous functions or by the means of teleoperation. Second, our team had a basic research interest in how to build up underwater response robots, working in a hazardous environment and how such robots are able to solve autonomously and effectively, complex tasks.

\section{Standardization and Testing}

The National Institute of Standards and Technology (NIST) has a strong reputation in standardization and testing in various domains. NIST also deals with complex cases, such as the evaluation of robotic platforms dedicated to search and hazmat operations. NIST's Robotics Test Facility - Building 207 - at the Gaithersburg campus, hosts a large number of robot test systems and artifacts (aka "props"), which are designed to be abstract representations of the targeted environments and tasks. The main mission of the facility is to foster the manufacturing and the deployment of advanced robotic systems through the development of performance testing methods (benchmarks), measurement capabilities and standards. Their work includes the assessment of joined sensors, intelligent behaviors, open-architecture controllers and high-fidelity simulation tools, summarized in the DHS NIST ASTM Robot Test Methods [2, 3].

The performance evaluation of mobile response robots has the following areas:

- Collaboration

- Autonomy

- Mapping and Planning 
- Knowledge Representation

- Sensory Perception

- Human-Robot Interaction

- Locomotion.

Intelligent response robots utilize a variety of sensors that include actuators, navigation and driving systems and communication systems. Just like any other typical robot, there is a need for mission-specific devices/packages controlled by an intelligent controller/ remote teleoperation.

For underwater response robots, autonomy is a common requirement. The survivability of the robot in an ever-changing, harsh environment depends on accurate situational and environmental awareness, based on reliable sensor data acquisition, data fusion, data evaluation and behavior generation (decision support).

Our proposed addition to the existing testing methods focuses on the temporal variability of the environment. In most cases, the robots are tested only for static scenarios. Such tests can hardly grasp how a response robot is able to accommodate to a new, suddenly changing environmental condition. Static terrain mapping can be misleading, if the environment is changing over time drastically (e.g., when a building fire spreads out, parts of a building collapse, or a boat is sinking).

\subsection{Proposed Additional Response Robot Testing Parameter Groups}

Our proposed two parameter groups, to assess the adaptability to the dynamically changing environment (temporal awareness) of a robot are the following:

- Temporal resolution (sensing/sampling frequency)

In many scenarios, the sampling frequency is an important factor. A good and simple example here is the real-time image acquisition, where fast moving objects are hardly recognizable if the frame rate is not high enough.

- Information aging speed

In a rapidly changing environment, the acquired data for data fusion, data evaluation and decision support can become outdated within a short time. Old and inaccurate data cause wrong situational (environment, location, etc.) awareness, and can introduce less effective behaviors than just using pure blind guessing. 


\section{Underwater Response Robot Testing Methods}

The test framework set up by NIST for unmanned underwater robots consists of various tasks [4]. In this paper, we are focusing on five selected domains from the aforementioned set:
1) Inspection/Station Keeping
2) Rope Cutting
3) Hooking
4) Soft Grab
5) Closing a valve

To accomplish these tasks, the manoeuvrability of a robot under trial should be precise and fine-tuned. Furthermore, smooth process controllers are needed for accurate positioning and depth tracking. The following examples are taken from tasks captured by a camera of an AUV during a NIST test execution.

\subsection{Inspection/Station Keeping}

This test measures the position keeping and the inspection capability of an AUV. During the task execution, there are various disruptive conditions, such as, turbidity or current. In order to compensate for these disturbances, typically, an underwater camera is installed on the robot. The objective of this task is to inspect cylinders on an underwater wall, and count the number of black lines placed in them. This translates into the thorough inspection of underwater areas. The precise position control is needed to solve this NIST task, because the cylinders are small, and the lines are only visible from a certain angle, thus better station-keeping capability is a major advantage. Fig. 1a shows the arrangement of the actual cylinders during a test round with an AUV.

\subsection{Rope Cutting}

In the second test case, the robots should clear an area enclosed by ropes. This method measures the cutting and targeting capability of the robot using different materials. The ropes are placed in different orientations. To solve this task, a cutting tool needs to be installed on the AUV. It has to be stable and sharp enough to cut through the thick, wet ropes. Fig. $1 \mathrm{~b}$ shows an example structure of the ropes. 

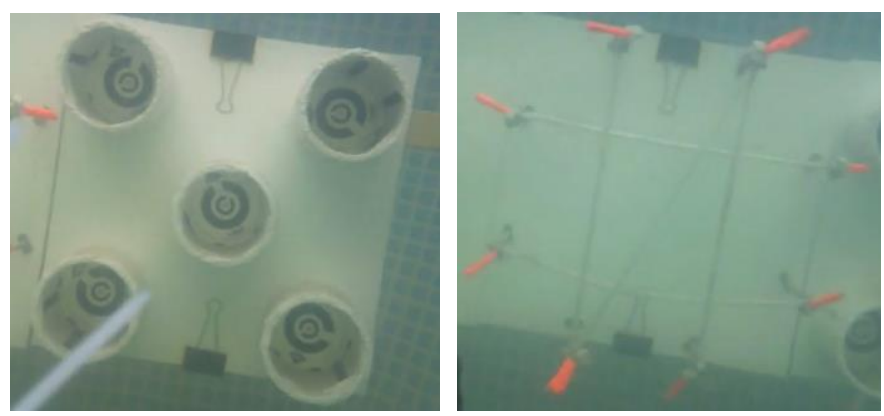

Figure $1 \mathrm{a}-\mathrm{b}$

Examples of the NIST underwater response robot capability tests a) The structure of the cylinders to be approached and explores

b) Rope structure to be cut by an AUV

\subsection{Hooking}

In the third test, the AUV should deploy a carabineer to the selected object, which is one of the loops placed in different directions. The complete object consists of 5 U-bolts, arranged in different orientations, thus the orientation of the carabineer is very important. Fig. 2a shows the structure of the carabineer holder.

\subsection{Soft Grab}

The fourth test is similar to the third; however, in this case, the robot should deploy an alligator clip on a soft target. The difficulty in this task is that the target keeps moving, driven by the currents and other conditions, therefore the AUV control methods must be much more sophisticated. Furthermore, precise positioning of the clip is required. Fig. $2 \mathrm{~b}$ shows the soft target and the clip.
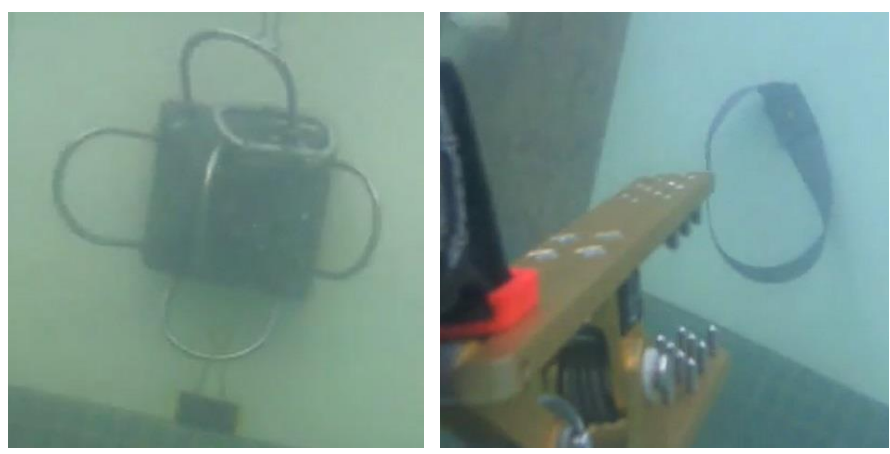

Figure $2 \mathrm{a}-\mathrm{b}$

a) AUV test case 3 , the structure of the carabineer holder

b) The fabric strap and the clip 


\subsection{Closing a Valve}

The fifth task is about closing and opening a valve as presented in Fig. 3. The object is set up in a rotatable way, and the AUV has to rotate the tap of the valve $90^{\circ}$ to the left and then to the right. To achieve this task, precise control of the robot is indispensable, because the AUV should maneuver up/down, left/right and forward/backward along a curved path.

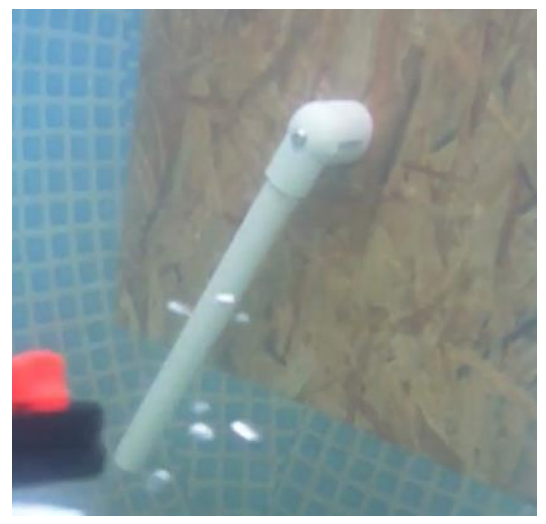

Figure 3

The mock of an underwater valve to be closed by an AUV

\section{Available Hardware and Software Components}

As a solid AUV platform to pass the NIST tests with, we employed a Sparus II lightweight hovering vehicle with mission-specific payload area and efficient hydrodynamics for long autonomy in shallow water (200 meters). The Sparus was originally developed at the University of Girona [5]. The AUV is torpedo-shaped, and has a built-in computer with an Intel Core i7 processor, 4 GB RAM, a 250 GB $\mathrm{SSD}$, and is equipped with a $1.5 \mathrm{kWh}$ battery (providing up to 8 hours autonomous navigation ${ }^{1}$. The Sparus II is shown in Fig. 4. It has 3 motors for underwater locomotion: one motor is for depth control and two are for maneuvering. On the software side, the system is based on the Robot Operating System (ROS), and has an additional software package named COLA2, which enables the hardware to use the integrated complex sensor and actuator systems. We have used this basic package and created our own software packages, for autonomous navigation and teleoperation. The Sparus is a very capable platform for developing an advanced AUV.

1 cirs.udg.edu/auvs-technology/auvs/sparus-ii-auv/ 

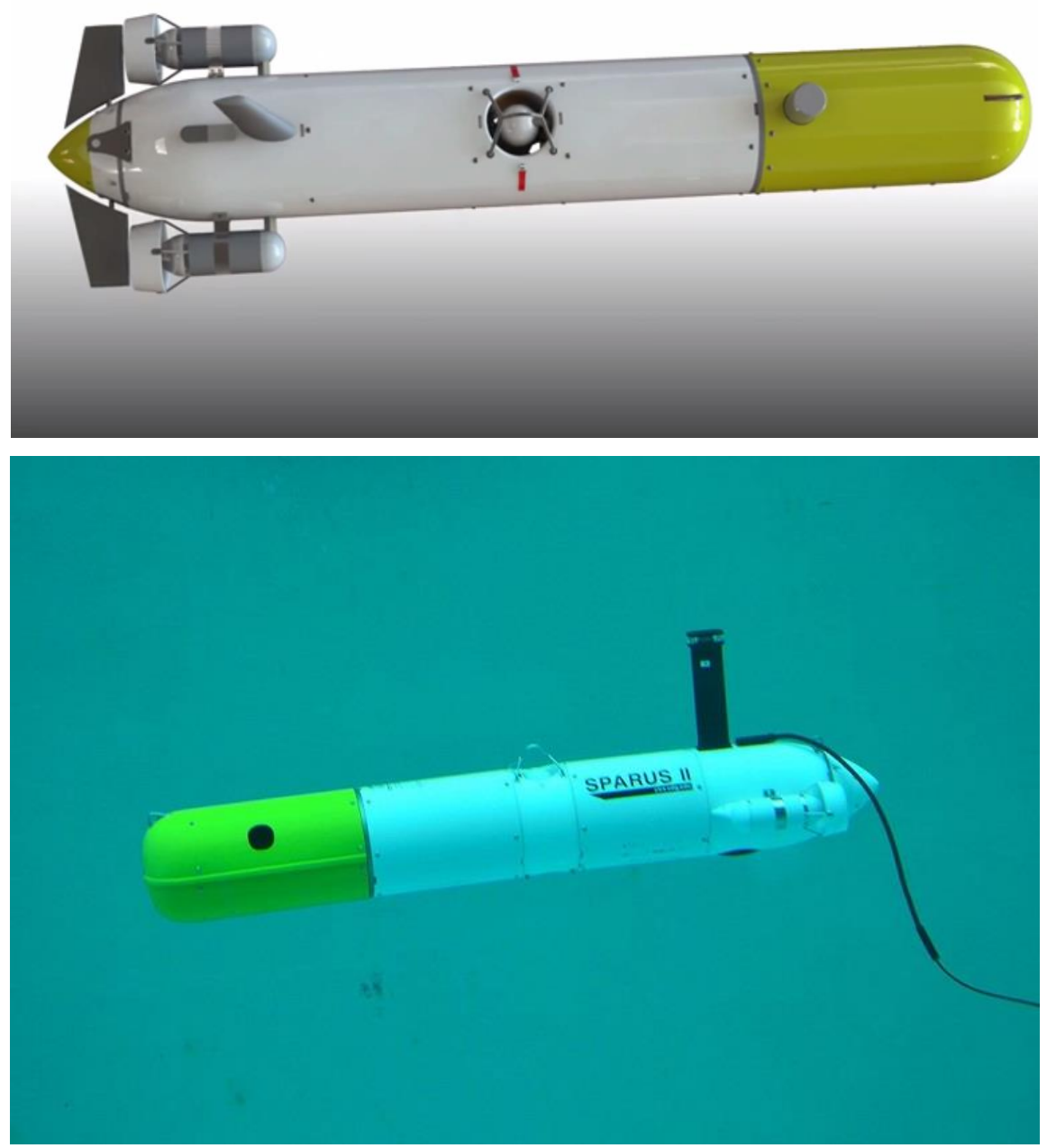

Figure 4

The Sparus II AUV platform developed at the University of Girona (Photo credit: University of Girona) 


\section{Extensions to the AUV Platform}

We designed two special hardware components to complete the NIST tests. The first is a waterproof cover for the CCD camera. It is indispensable for performing teleoperation tasks with the robot and the mobile manipulator later equipped on the Sparus. The other components were two waterproof covers for the servos, enabling us to build an underwater mobile 2+1 Degree of Freedom (DoF) manipulator for task execution.

\subsection{Waterproof Camera Cover}

We used a Microsoft LifeCam Cinema HD USB web camera (Fig. 5) to provide high quality real-time video streaming. The first step was making a waterproof cover for the web camera. The biggest challenge was presented by the external pressure, as the comparable water pressure is about 2 bars $(200 \mathrm{kPa})$ at 10 meters below the surface. This means $2 \mathrm{~kg}$ weight on every $1 \mathrm{~cm}^{2}$. The other difficulty was the corrosive effect of the sea water, when we used plastic materials (Plexiglas, thermosetting plastic) to manufacture the cover.

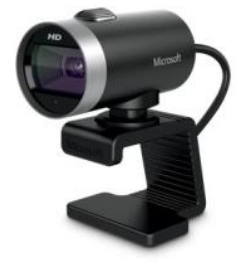

Figure 5

Microsoft LifeCam Cinema HD USB web camera

\subsection{Waterproof Servo Cover}

To create a mobile manipulator, we employed model RC servos, like the ones used in model boats and cars. These servos are not waterproof, therefore, we designed a custom cover for each of them. The case is compatible with all of the standard sized servos that can be found in commercial distribution. We built a 2+1 DoF robot manipulator with simple kinematics (Fig. 6) from these servos, where each DoF is providing an orientation, while another 3 DoF were derived from the AUV's ability for positioning. 


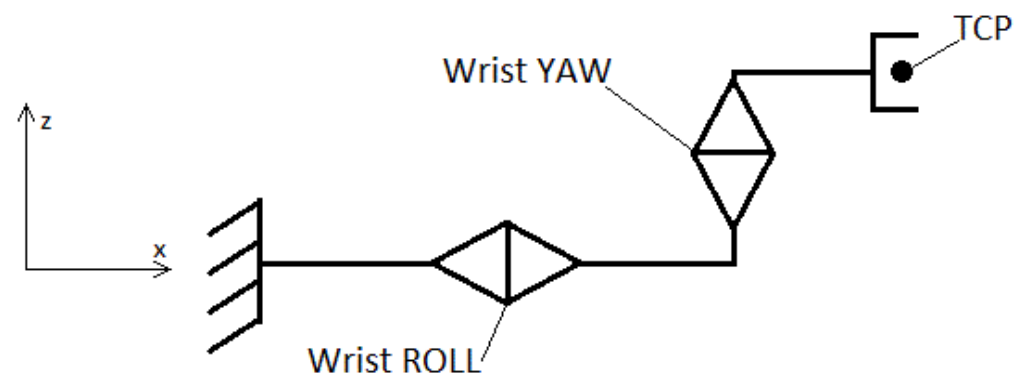

Figure 6

Kinematic structure of the underwater arm designed for the AUV

\section{Hardware Implementation}

\subsection{Design and Manufacturing of the Waterproof Camera Cover}

The components of the cover were designed using the SolidWorks modeling software. Fig. 7 shows the exploded 3D CAD model of the cover.

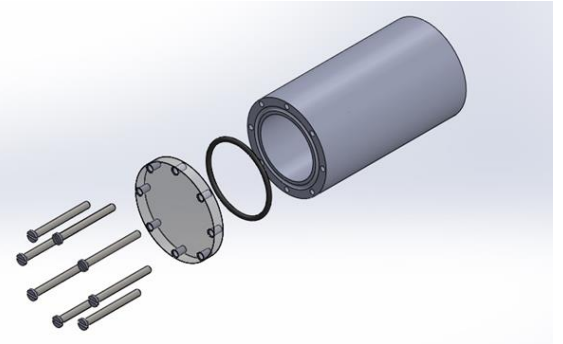

Figure 7

3D CAD model of the waterproof camera cover for the AUV

The base of the cover was made of thermosetting plastic using a lathe. In the front, there is a lid, made of water-clean Plexiglas, and the hermetic seal is provided by an O-ring. The lid is secured by eight M3 screws, and the outlet of the USB cable is insulated with epoxy glue and silicone rubber. Fig. 8 shows the cover with an installed camera. 


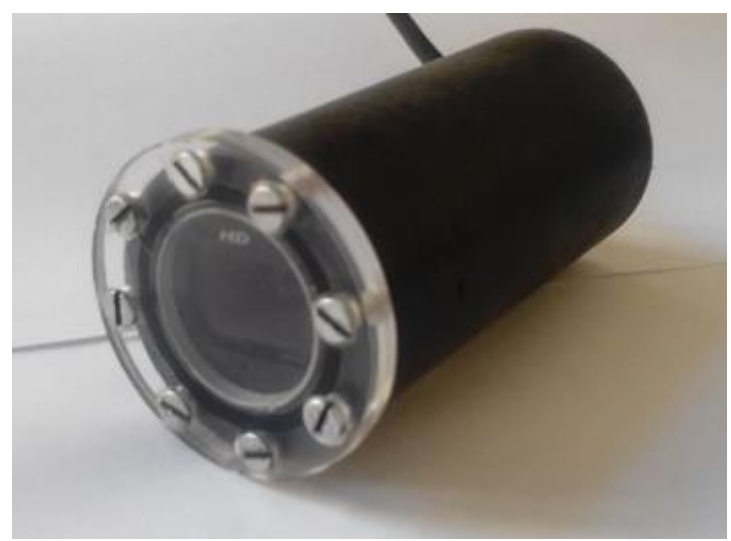

Figure 8

The AUV camera fit into its waterproof cover

\subsection{CAD/CAM of the Waterproof Servo Cover}

The servo cover was also designed in SolidWorks. This cover is composed of two parts: the top contains two ball bearings for holding the drive axle stable. The hermetic seal is provided by a lip seal. The drive axle and the axle of the servo are connected by coupling. In the bottom part, there is an outlet for a cable. It is also insulated by epoxy glue and silicone rubber. Fig. 9 shows the 3D CAD model of the cover.
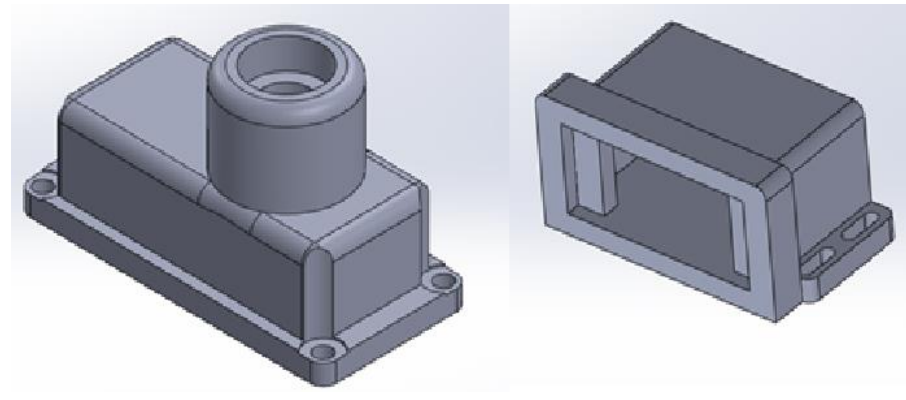

Figure 9

3D CAD model of the waterproof servo cover 


\section{Software for Teleoperation}

The implemented teleoperation solution in the AUV is a ROS node that communicates in two directions (Fig. 10). On the one hand, it reads values from the "/joy" topic of the COLA2 framework in every $50 \mathrm{~ms}$. The values are the states of the buttons of the integrated Xbox 360 controller. The left and right triggers and arms are used on the controller, thus the values of these control events are sent to the thrusters and the Arduino Mega microcontroller.

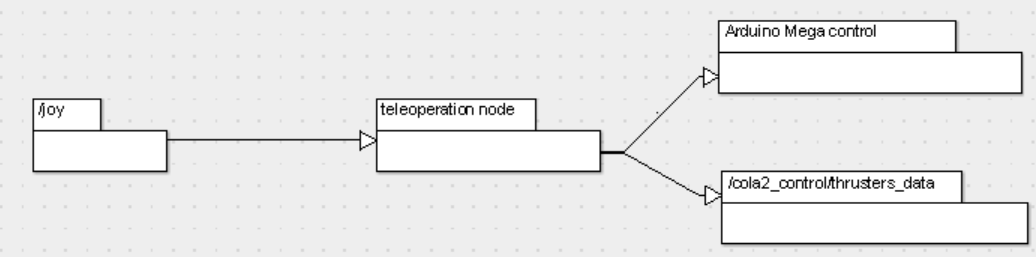

Figure 10

Teleoperation ROS node architecture for the AUV

With the vertical movements of the triggers and arms, the AUV is directly controlled. The game controller posts into the "/joy" COLA2 topic a value between -1 and 1 every time when some status change occurs. Fortunately, the thrusters of the AUV can be operated by values between the same intervals, so it is not justified to map the value between other intervals. These values can be forwarded directly to the "/cola2_control/thrusters_data" ROS topic, where the control of the thrusters is solved. Because of the noisy signals of the Xbox 360 controller, the values of the arms and triggers between 0 and 0.3 are considered as 0 . A forwarded 1 means that the thruster should work with $100 \%$ performance, -1 is the opposite, $-0.3-0.3$ means that the thruster is stopped. This operates on a similar principle in the case of the servos. The vertical movement of the left trigger, of the joystick, results in the AUV moving forward or backward. On the other hand, the right trigger of the joystick results in the device turning left or right. If the operator wants to turn left or right, the value of the left and right trigger will be sent to the "/cola2_control/thrusters" data. The left thruster will receive the value, while the right thruster will receive the value with the opposite sign. The maximum performance output of the thrusters is not enabled, and automatically degraded to a safe performance output value by the software. 


\section{Underwater Manipulation}

This low-cost underwater manipulator, with three DoF, required additional software and hardware components for the AUV to work. An Arduino Mega 2650 microcontroller was used for direct control, and a Xbox 360 game controller enables the human operator to drive the manipulator indirectly. The ROS connected both control units. During teleoperation, the game controller was remapped to enable the smooth 2 DoF movements, of the manipulator. The left arm of the game controller defined the vertical movements. (This first servo is attached to the AUV). The right arm of the game controller defines the movement of the second servo attached to the first one. After some tests, it was deduced that the arms of the Xbox 360 game controller returns a value between -1 and 1, so if the program is able to read this value every $50 \mathrm{~ms}$, it could move a servo up to 20 degrees per minute. This simple solution was robust enough to be used for servo control. According to our tests with a polling rate of 20 per second, a smooth underwater manipulation with sufficient precision is realized.

\section{Tests}

Some tests have been carried out after the realization of the waterproof cases. The first test environment was a pressure chamber with 10 bars, where all tests were successful. After this, we attached it to the AUV at a temporary location. For different kind of tests, a pool was set up outside the lab, filled with fresh water. It had the dimensions of $4 \times 2 \times 2$ meters ( $\mathrm{L} \times \mathrm{W} \times \mathrm{H}$ ). The tools created by the team were left in the pool with the AUV to decide how well they could stand up against the water. The next test environment was also a pool. It was set up in the euRathlon 2015 competition (http://eurathlon.eu/) for test purposes, yet filled up with sea water. One of our camera cases was slightly damaged by the salty water, but destroyed, during the competition, so we continued the NIST tests. The last test environment was the euRathlon 2015 competition (S1 and S2 session) where we had to use our device in five meters of depth, performing some of the NIST tests.

\section{Lessons Learned}

The outdoor euRathlon 2015 competition and the NIST trials were the ultimate testing environments for our AUV. Both the developers and the response robot had to cope with the real-world scenario. It was a physical challenge that brought both human and machine to their limits. The very first problem was the difference between the software based simulation environment and reality was that we were 
able to realize during the firsts tests in the Lupa lake in Hungary, where a lot of time was spent balancing the AUV. Without proper balance, autonomous navigation algorithms and teleoperation solutions cannot work properly. Another serious problem was the inappropriate choice of materials of some of the 3D printed components. Certain kinds of materials can be damaged by the sea water and the team did experience this during the trials in fresh water. The first $3 \mathrm{D}$ models were printed with PLA instead of ABS, and salt water has an effective degenerative effect on PLA. Furthermore, it was easy to integrate the Xbox 360 joystick into the AUV system, but it was very difficult to achieve a smooth control of the thrusters with teleoperation, which was definitely needed for the NIST tests.

There was a special failure of the system that we realized during the tests. Every time we wanted to control the thrusters at a high RPM, the USB web camera was detached by the operation system that runs on the AUV, so the camera was not able to support our solutions and the system. The problem turned out to be the high pulse generated by the thrusters, the metal body of the AUV, and the inadequate insulation of the cables. Once the cables were properly insulated, this failure disappeared instantly.

\section{Conclusions}

This paper describes our work in AUV response robot testing, from two aspects: we proposed additional testing methods for AUV capability assessment. Here, two parameter groups were identified to assess the adaptability to a dynamically changing environment (temporal awareness) of the robots. These are the temporal resolution (sensing/sampling frequency) and Information aging speed. Further, we detailed how a Sparus II platform based AUV can be enhanced to pass the existing underwater response robot tests defined by National Institute of Standards and Technology (NIST).We developed software and hardware components to extend the capabilities of the AUV platform: the additional software components were indispensable for precise navigation, position holding and teleoperation. Beyond this, we integrated an Xbox 360 game controller, a self-made waterproof manipulator arm and camera. The sensor provided the required visual data for teleoperation, the game controller and the actuator enabled the smooth operation and control of the manipulator joints. The outdoor euRathlon 2015 competition and the NIST tests were the real field trials in a physical environment for our AUV system. The project provided us with massive opportunities to find and successfully resolve major, "real-world" engineering challenges.

\section{Acknowledgement}

Authors would like to thank NATO Centre for Maritime Research and Organization (CMRE) for the opportunity to access and use the Sparus II AUV during the euRathlon competition, and also the friendly support of NIST and University of Girona (UdG). The financial support of this work was from the University Research and Innovation Center, Óbuda University, Hungary (URIC/EKIK). 


\section{References}

[1] W. Dirk, D. R. Edgington and C. Koch. "Detection and Tracking of Objects in Underwater Video." In Proc. of the IEEE Computer Vision and Pattern Recognition. CVPR 2004, Vol. 1, pp. I-544, 2004

[2] Underwater Robotics Research Centre (CIRS), "Design of SPARUS II AUV," Technical Report. cirs.udg.edu/wp-content/uploads/2014/05/ /SPARUS_II_design.pdf, 2014

[3] A. Jacoff, E. Messina, H.-M. Huang, A. Virts, A. Downs, R. Norcross, R. Sheh; "Guide for Evaluating, Purchasing, and Training with Response Robots using DHS-NIST-ASTM International Standard Test Methods"; NIST Standard Test Methods For Response Robots; ASTM International Standards Committee on Homeland Security Applications (E54.08.01). http://www.nist.gov/el/isd/ks/upload/DHS_NIST_ASTM_Robot_Test_Met hods-2.pdf, 2014

[4] A. Jacoff, K. Saidi, R. von Loewenfeldt, Y. Koibuchi; "Development of Standard Test Methods for Evaluation of ROV/AUV Performance for Emergency Response Applications", in Proc. of the $16^{\text {th }}$ MTS/IEEE OCEANS, Washington DC, 2015

[5] M. Carreras, C. Candela, D. Ribas, N. Palomeras, L. Magí, A. Mallios, E. Vidal, È. Vidal and P. Ridao; "Testing SPARUS II AUV, an open platform for industrial, scientific and academic applications", $6^{\text {th }}$ Intl. Workshop On Marine Technology, Martech 2015, Cartagena, pp. 106-109, ISBN: 978-84-608-1708-6, 2015 